\title{
Meson-production experiments at COSY-Jülich
}

\author{
M. Büscher ${ }^{1 ; 1)}$ \\ 1 (Institute für Kernphysik and Jülich Center for Hadron Physics, Forschungszentrum Jülich, 52425 Jülich Germany)
}

\begin{abstract}
Selected results from experiments at COSY-Jülich are presented: an attempt to measure the mass of the $\eta$ meson with high precision (ANKE facility), first steps towards the detection of rare $\eta$ decays (WASA), and several measurements of $K \bar{K}$-pair production (ANKE, COSY-11, MOMO).
\end{abstract}

Key words Meson production

PACS 14.40.Cs Other mesons with $S=C=0$, mass $<2.5 \mathrm{GeV}$

\section{Introduction}

The Cooler Synchrotron and storage ring COSY ${ }^{[1]}$ of the Forschungszentrum Jülich delivers unpolarized and polarized beams of protons and deuterons with momenta up to $3.7 \mathrm{GeV} / \mathrm{c}$. Currently, these are utilized at three large experimental facilities, ANKE and WASA at internal target stations and COSY-TOF at the extracted beam.

- ANKE (Apparatus for Studies of Nucleon and Kaon Ejectiles) is a large acceptance forward magnetic spectrometer ${ }^{[2]}$. The central dipole is movable to adjust the momenta of the detected particles independent of the beam momentum. Using hydrogen or deuterium cluster targets ${ }^{\underline{\underline{3}}}$, close-to-threshold reactions on protons or neutrons can be measured. In addition, a polarized internal target with a storage cell can be used. The ANKE detectors have been optimized for charged kaon detection ${ }^{[4]}$ which allow one to identify $K^{+} K^{-}$pairs ${ }^{[\underline{5}]}$ in coincidence with fast forward going protons and deuterons $\underline{\underline{6}}$.

- TOF (Time Of Flight) is a non-magnetic spectrometer combining excellent tracking capabilities with large acceptance and full azimuthal symmetry allowing to measure complete Dalitz $\operatorname{plots}^{[\underline{\underline{z}}}$. TOF is optimized for final states with

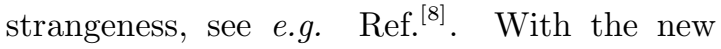
straw tube tracking system (STT), TOF will have a significantly improved mass resolution and reconstruction efficiency.
- WASA (Wide Angle Shower Apparatus), is an almost $4 \pi$ spectrometer for neutral and charged particles. It comprises an electromagnetic calorimeter, a very thin superconducting solenoid, inner and forward trigger and tracking detectors, and a frozen-pellet target. WASA was build at TSL Uppsala and it was used there until 2005 at the CELSIUS ring ${ }^{[9]}$. The system was transferred to COSY in 2005 and it was operational already after one year. One of the main goals for WASA at COSY is the study of rare $\eta$-meson decays involving photons and electrons ${ }^{[10]}$.

Here we report on selected ongoing measurements carried out at the ANKE and WASA facilities and on data from the decommissioned COSY-11 and MOMO experiments. For other results from COSY see the contributions to this conference by M. Bashkanov (two-pion production) and H. Machner ( $\eta$ mesons in matter).

\section{Precision measurement of the $\eta$ mass}

Measurements of the $\eta$-meson mass at different experimental facilities over the last decade have resulted in remarkably precise results. However, these differ by up to $500 \mathrm{keV} / c^{2}$, which is more than 8 standard deviations of the quoted individual experimental uncertainties. As a consequence the PDG tables no longer consider experiments based on the identification of the $\eta$ as a missing-mass peak of a hadronic reaction. In order to check whether there is an intrinsic 
problem in such experiments, a refined measurement of the $d p \rightarrow{ }^{3} \mathrm{He} \eta$ reaction has been carried out at COSY.

After producing the $\eta$ mesons through the $d p \rightarrow$ ${ }^{3} \mathrm{He} \eta$ reaction, the ${ }^{3} \mathrm{He}$ is detected with the ANKE forward detectors ${ }^{[6]}$. Reactions with the production of an $\eta$ meson can then be unambiguously identified from a missing-mass criterion ${ }^{[11]}$. Due to the simple two-body kinematics of the ${ }^{3} \mathrm{He} \eta$ final state, the $\eta$ mass can be determined through a thorough reconstruction of the size of the ${ }^{3} \mathrm{He}$ kinematic ellipse. This, in turn, relies on a precise identification of the reaction threshold and an accurate measurement of the associated beam momentum ${ }^{[12]}$.

The measurements were carried out at thirteen beam energies in the range $1<Q<10 \mathrm{MeV}$ as well as $Q=-5 \mathrm{MeV}$ for background studies $\underline{\underline{12]}}$. To determine the $\eta$ mass with a precision that is competitive to other state-of-the-art measurements, i.e. $\Delta m_{\eta}<50 \mathrm{keV} / c^{2[13]}$, the associated beam momenta have to be fixed with an accuracy of $\Delta p / p<10^{-4}$. This can be achieved via the precise measurement of the frequency of depolarizing resonances $f_{r}$ and of the beam revolution frequency $f_{0}$. The usefulness of the technique relies on the fact that frequencies can be routinely measured to $10^{-5}$.

From the measured frequencies $f_{r}$ and $f_{0}$ the kinematical factor $\gamma=E / m c^{2}$, and hence the beam momentum $p$, can be deduced:

$$
\begin{aligned}
\gamma & =\frac{1}{G_{d}}\left(\frac{f_{r}}{f_{0}}-1\right) \\
p & =m_{d} \beta \gamma=m_{d} \sqrt{\gamma^{2}-1} .
\end{aligned}
$$

Here $E$ denotes the deuteron total energy, $m_{d}$ the deuteron mass and $G_{d}=(g-2) / 2$ is the gyromagnetic anomaly of the deuteron, where $g$ is the gyromagnetic factor.

The spins of the polarized deuteron beam particles precess around the normal to the plane of the machine, which is generally horizontal. The spin can be perturbed by the application of a horizontal $r f$ magnetic field from, for example, a solenoid. The beam depolarizes when the frequency of the externally applied field coincides with that of the spin precession in the ring. An example of a spin-resonance spectrum is shown in Fig. 1. Far away from the spin resonance at $1.0116 \mathrm{MHz}$ and $1.0120 \mathrm{MHz}$, a high degree of polarization was measured using the EDDA detector ${ }^{[14]}$ as a beam polarimeter. In contrast, when the solenoid frequency coincides with that of the spin precession $f_{r}$, the beam is maximally depolarized.

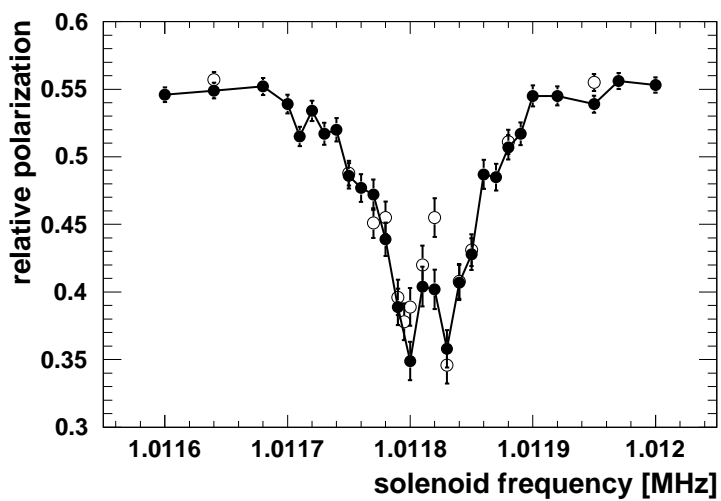

Fig. 1. Spin resonance measurements at one beam momentum (closed circles). The open symbols represent results obtained for an extended cycle time, where the perturbing solenoid was switched on after $178 \mathrm{~s}$.

The beam revolution frequency $f_{0}$ was measured from the Schottky noise of the deuteron beam. The origin of this effect is the statistical distribution of the charged particles in the beam. This leads to random current fluctuations, which induce a voltage signal at a beam pick-up. The Fourier transform of this voltage-to-time signal by a spectrum analyzer delivers the frequency distribution around the harmonics of the revolution frequency of the beam.

The accuracies to which both of the frequencies are determined are dominated by systematic effects. The revolution frequency measured by the Schottky spectrum analyzer has an uncertainty of $\Delta f_{0}=6 \mathrm{~Hz}$, corresponding to one in the beam momentum of $50 \mathrm{keV} / c$. The error in the determination of the spin resonance arises from the small variations of the orbit length and $\Delta f_{r}=15 \mathrm{~Hz}$ corresponds to an uncertainty in the beam momentum of $164 \mathrm{keV} / c$. Because these systematic uncertainties are independent, they are added quadratically to give a total uncertainty $\Delta p / p \leqslant 6 \times 10^{-5}$, i.e., a precision of $170 \mathrm{keV} / c$ for beam momenta in the range of $3100-3200 \mathrm{MeV} / c$. This is over an order of magnitude better than ever reached before for a standard experiment in the COSY ring. This accuracy will ultimately allow the mass of the $\eta$ meson to be measured with a precision of $\Delta m_{\eta} \leqslant 50 \mathrm{keV} / c^{2}$. The remaining part of the data analysis is under way.

\section{Towards rare $\eta$ decays}

The initial stage of the $\eta$ meson decay experiments with WASA was carried out at CELSIUS using the $p p \rightarrow p p \eta$ and $p d \rightarrow{ }^{3} \mathrm{He} \eta$ reactions close to threshold. 
Results on the Dalitz plot density for the $\eta \rightarrow 3 \pi^{0}$ decay ${ }^{[15]}$ and on the branching ratios of some leptonic decay channels $\left.{ }^{[16}, 17\right]$ have been reported.

During the last two years, larger data samples have been collected at COSY on $\eta$ decays from $p p$ and $p d$ collisions. From a short production run in April 2007 120k events have been extracted for the $\eta \rightarrow 3 \pi^{0}$ Dalitz plot ${ }^{[18]}$. In the next 4 - and 8-week run periods in fall 2008 and fall 2009, $\eta$ decays were studied using the $p d \rightarrow{ }^{3} \mathrm{He} \eta$ reaction at a beam energy of $1 \mathrm{GeV}$. Unbiased data samples of $1.1 \times 10^{7}$ and $\sim 2 \times 10^{7} \eta$ meson decays were collected, respectively. In addition, a few shorter runs were also carried out with the aim of optimizing the conditions for $\eta$ meson production from $p p$ collisions.
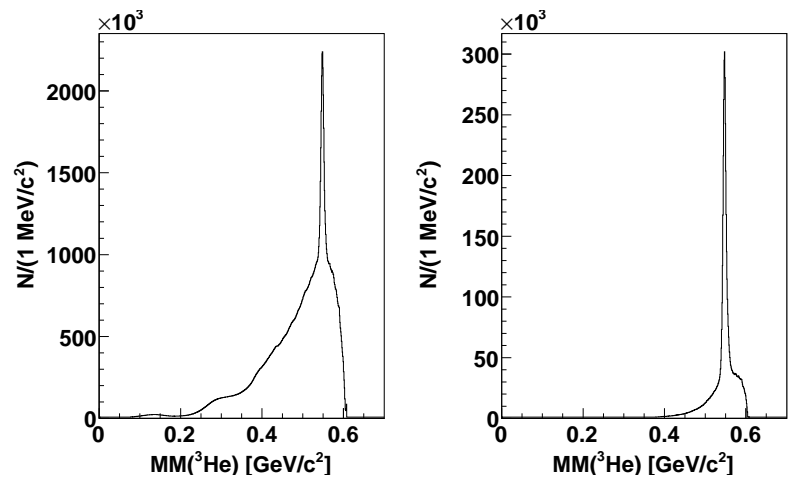

Fig. 2. Distribution of ${ }^{3} \mathrm{He}$ missing mass for the $p d \rightarrow{ }^{3} \mathrm{He} X$ reaction at $1.0 \mathrm{GeV}$. The trigger was based entirely on ${ }^{3} \mathrm{He}$ signal in the forward detector without any bias on the decay system. The plot shows all the data collected during the 2008 run period. (Left) data analysis based only on the forward detector track - there are about $1.1 \times 10^{7}$ events in the peak at the $\eta$ meson mass. (Right) in addition twophoton invariant masses $\geq 300 \mathrm{MeV} / \mathrm{c}^{2}$ have also been demanded.

The cross section of $p d \rightarrow{ }^{3} \mathrm{He} \eta$ reaction rises quickly and reaches a plateau value of $400 \mathrm{nb}$ already at $2 \mathrm{MeV}$ above the threshold. The requirement of a ${ }^{3} \mathrm{He}$ ion in the final state selects only a very tiny fraction of the total $p d$ cross section (about $0.1 \%$ ). The separation of ${ }^{3} \mathrm{He}$ from protons and deuterons is quite reliable and is implemented on the trigger level. When doing this, one obtains trigger rates at maximum luminosity that are well within the DAQ capabilities without imposing any additional constraints on the $\eta$ decay pattern.

The $p d \rightarrow{ }^{3} \mathrm{He} \eta$ reaction is tagged by identifying the ${ }^{3} \mathrm{He}$ particles in the WASA forward detector, which covers scattering angles from $3^{\circ}$ to $18^{\circ}$. Figure 2 shows the missing mass of the reconstructed ${ }^{3} \mathrm{He}$ at $1.0 \mathrm{GeV}$ beam energy. In the left panel all data collected in the $2008 p d$ run period are shown. In the right panel after selection of a particular decay channel.

The WASA detector was optimized for the measurement of electron-positron pairs and photons from the decays of $\pi^{0}$ and $\eta$ mesons. The performance of the detector can be checked by studying single Dalitz decays $\eta \rightarrow e^{+} e^{-} \gamma$ and $\pi^{0} \rightarrow e^{+} e^{-} \gamma$. The decays were observed in both $p p$ and $p d$ interactions. Figure 3 shows an example of the $\eta \rightarrow e^{+} e^{-} \gamma$ decay identification in the invariant mass of $e^{+} e^{-} \gamma$ from the $p d$ data. The data from both $p d$ and $p p$ runs should contain a few thousands of $\eta \rightarrow e^{+} e^{-} \gamma$ events.

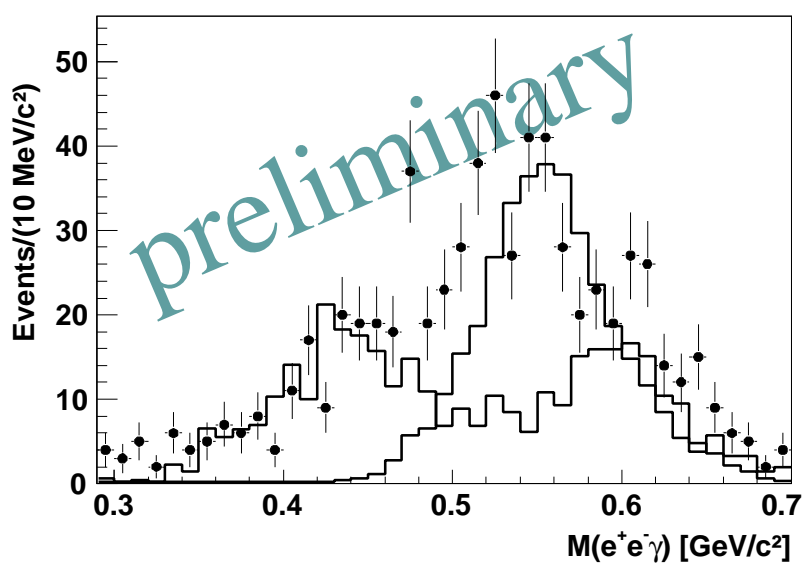

Fig. 3. Single Dalitz decay $\eta \rightarrow e^{+} e^{-} \gamma$ events for about $10 \%$ of the $2008 p d$ data. The histograms represent Monte Carlo simulations of the signal (solid line) and background (dashed line).

The decay $\eta \rightarrow \pi^{+} \pi^{-} e^{+} e^{-}$allows precise tests of the chiral anomaly through the comparison with existing ChPT calculations. A high statistics measurement of $\eta \rightarrow \pi^{+} \pi^{-} e^{+} e^{-}$will also provide constraints for a new kind of flavor-conserving CP violation by measuring the asymmetry of the dihedral angle between the pion- and electron planes. The present $\mathrm{PDG}$ value for the branching ratio of the $\eta \rightarrow \pi^{+} \pi^{-} e^{+} e^{-}$decay is $4.2 \times 10^{-4}$.

The status of the analysis of the $\eta \rightarrow \pi^{+} \pi^{-} e^{+} e^{-}$ decay is shown in Fig. 4. A clear signature with about 300 events of the $\eta \rightarrow \pi^{+} \pi^{-} e^{+} e^{-}$decay is seen in the ${ }^{3} \mathrm{He}$ missing mass. The line represents a polynomial fit to the direct pion production reactions. According to the Monte Carlo simulations, $50 \%$ of the events in the $\eta$ mass peak are from $\eta \rightarrow \pi^{+} \pi^{-} e^{+} e^{-}$. 


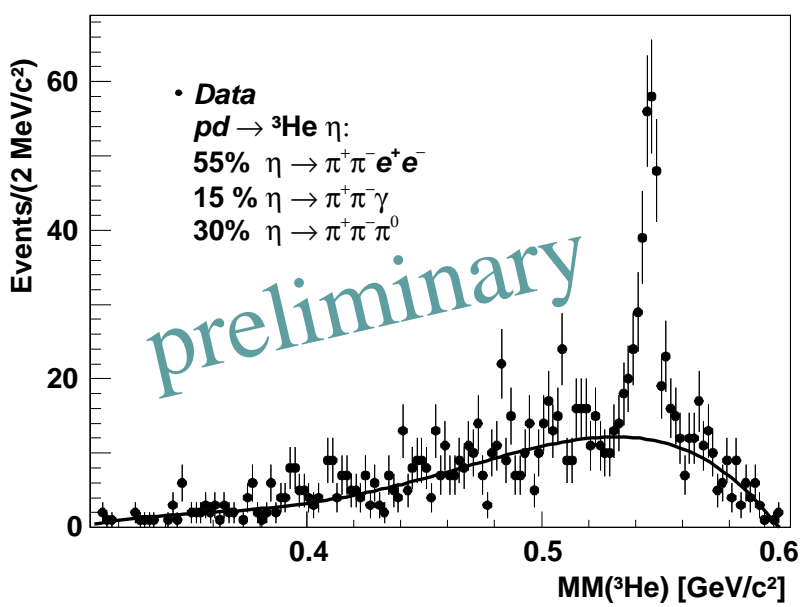

Fig. 4. Missing mass of ${ }^{3} \mathrm{He}$ for events compatible with $\eta \rightarrow \pi^{+} \pi^{-} e^{+} e^{-}$. The line is a polynomial fit to the background.

The major background contributions come from the $\eta \rightarrow \pi^{+} \pi^{-} \gamma$ decay, with $\gamma$ conversion in the beam tube. Additional background stems from $\eta \rightarrow$ $\pi^{+} \pi^{-} \pi^{0}$, due to internal (Dalitz decay of the $\pi^{0}$ ) and external conversion of one of the photons from $\pi^{0} \rightarrow \gamma \gamma$ decays. At present, the analysis does not include any vertex reconstruction which will help to suppress the background from external conversion. The remaining background will be dominated by $\eta \rightarrow \pi^{+} \pi^{-} \pi_{\text {Dalitz }}^{0}$ and can be further reduced by improving the detection sensitivity for the additional photon. The overall reconstruction efficiency is limited by requesting the suppression of the background and is $5-10 \%$.

The measurement of double Dalitz $\eta$ decays, $\eta \rightarrow$ $e^{+} e^{-} e^{+} e^{-}$allows the study of the $\eta$ meson form factor for two timelike virtual photons. In the 2008 pd data, 15 candidate events have been identified.

The $\eta$ decay into $\pi^{0} e^{+} e^{-}$is forbidden from proceeding via an intermediate $\pi^{0} \gamma^{\star}$ state due to Cconservation. The present upper limit for the branching ratio is $\leq 4 \times 10^{-5}$. The upper limit would correspond to a maximum of 400 events in the $2008 \mathrm{pd}$ run period. No signal is observed in the data. Although the analysis is in an early stage, our sensitivity is already close to the present upper limit. A refined analysis including a full kinematic fit will further suppress the contributions from background channels and enhance the sensitivity.

The dominant mechanism for $\mathcal{P} \rightarrow \ell^{+} \ell^{-}$decays within the Standard Model is a process involving two virtual photons and this is additionally suppressed by helicity conservation. Recently the interest in the decays was revived due to an observed excess for $\pi^{0} \rightarrow$ $e^{+} e^{-}$decay branching ratio ${ }^{[19]}$. For the $\eta \rightarrow e^{+} e^{-}$ decay the best experimental limit $\left(2.7 \times 10^{-5}\right)$ comes from CELSIUS/WASA ${ }^{[17]}$.

Due to WASA's calorimeter and MDC particle flux limitations, the maximal rate of $\eta$ mesons from the $p d \rightarrow{ }^{3} \mathrm{He} \eta$ reaction is about $10 \eta$ events/s. Therefore the reaction can only be used for studies of notso-rare $\eta$ decays. Further progress towards the measurement of rare $\eta$ decays can only be made by focusing on the $p p \rightarrow p p \eta$ production reaction. The reaction has $10-20$ times larger cross section $(10 \mu \mathrm{b}$ at $1.4 \mathrm{GeV}$ ) and the inclusive $p p$ cross section is two times lower than for $p d$ interactions. Therefore the $\eta$ meson yield will be higher for a given luminosity and, in addition, one can use up to two times higher luminosity. A first production beam time of 8 weeks is scheduled for spring 2010. The goal is to observe about $10^{10} \eta$ decays per year from then on.

\section{Kaon-pair production}

Over the last decade, exhaustive measurements on kaon-pair production have been carried out at several COSY facilities. Values of total and differential cross sections are now available for various isospin configurations of the entrance and exit channels, $c f$. Fig. 5 ,

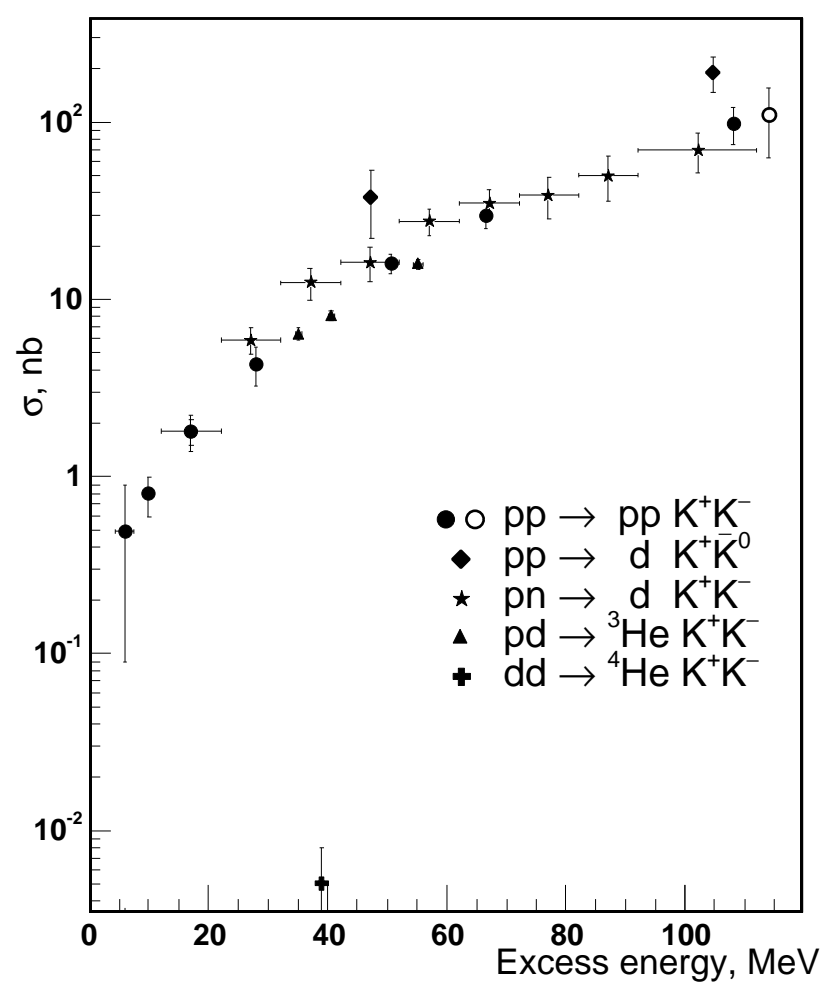

Fig. 5. World data set on total cross sections for kaon-pair production. Closed symbols denote results from COSY for the $p p \rightarrow$ $p p K^{+} K^{-}, p p \rightarrow d K^{+} \bar{K}^{0}, p n \rightarrow d K^{+} K^{-}$, $p d \rightarrow{ }^{3} \mathrm{He} K^{+} K^{-}$, and $d d \rightarrow{ }^{4} \mathrm{He} K^{+} K^{-}$reactions. 
The $p p \rightarrow p p K^{+} K^{-}$reaction has been studied by the COSY-11 $\underline{[20}, \underline{21]}$ and ANKE $\underline{[22,}$,23] collaborations for excess energies $\varepsilon$ ranging from 3 to $108 \mathrm{MeV}$. Also the isospin-related $d K^{+} \bar{K}^{0}$ exit channel has been measured at ANKE for $\varepsilon=47$ and $105 \mathrm{MeV}^{[24}, \underline{25]}$. The $p n \rightarrow d K^{+} K^{-}$reaction has been investigated at $\mathrm{ANKE}^{[\underline{[26}, \underline{27}]}$ using a deuterium cluster-jet as an effective neutron target. In such measurements, the momentum of the non-observed proton spectator, and thus the excess energy $\varepsilon_{K \bar{K}}=17-102 \mathrm{MeV}$, is reconstructed from the four-momenta of the remaining detected particles.

These $p N \rightarrow(2 N) K \bar{K}$ data have been analyzed in terms of the relevant final-state interactions (FSIs). It turned out that - besides the well known interaction of the two outgoing nucleons - there is a delicate interplay between the $K \bar{K}$ and $\bar{K} N$ FSIs, see e.g. Ref. ${ }^{[28]}$. The $K^{+} N$ interaction is generally believed to be small and has been neglected in all analyses.

The produced $\bar{K} N$ systems may provide valuable information about the much debated antikaonnucleon interaction strength and, possibly, about intermediate hyperon states such as the $\Sigma(1385)$ or the $\Lambda(1405)$. In fact, for the $p p \rightarrow d K^{+} \bar{K}^{0}$ reaction the $\bar{K} d$ pairs are found preferentially at small invariant masses; the partial wave decomposition reveals a $\bar{K} d$ $S$-wave enhancement as compared to $K^{+} d^{[28]}$. The same low-mass enhancement has also been found in the $p n \rightarrow d K^{+} K^{-}$data ${ }^{[27]}$. This has been interpreted as evidence for a strong attractive $\bar{K} d$ FSI and the $\bar{K}^{0} d$ data are best fit with a scattering length of $\left|a_{\bar{K} d}\right|=1.5 \mathrm{fm}^{[28]}$. A similar effect has been observed for the $p p \rightarrow p p K^{+} K^{-}$data. Here the enhancement at low $K^{-} p$ and $K^{-} p p$ masses is best fit with a $\bar{K} p$ scattering length of $a_{\bar{K} p}=(0+1.5 i) \mathrm{fm}^{\underline{[23]}}$.

The $K \bar{K}$ interaction may, in principle, be driven by the light scalar mesons, $a_{0} / f_{0}(980)$, as well as the $\phi(1020)$. In fact, all data sets with $(K \bar{K})^{0}$ pairs reveal a clear $\phi(1020)$ signal when its production is energetically allowed. The $a_{0} / f_{0}(980)$ have widths of about $50-100 \mathrm{MeV}$, i.e. much larger than that of the $\phi(1020)$. They are thus harder to be found in the $K \bar{K}$ mass distributions, in particular at COSY energies where the excess energy is limited to roughly $100 \mathrm{MeV}$. Consequently, there is only indirect evidence for scalar-meson production from the partialwave decompositions of the $p p \rightarrow p p K^{+} K^{-}$and $p p \rightarrow$ $d K^{+} \bar{K}^{0}$ data. These reveal an $S$-wave dominance in the $K \bar{K}$ systems $\underline{\underline{22}}, \underline{24}, \underline{25]}$, which may be interpreted in terms of kaon production via the $a_{0}^{0} / f_{0}$ and $a_{0}^{+}$ channels, respectively.
On top of the above discussed FSIs there is an enhancement at low $K \bar{K}$ masses, i.e. between the $K^{+} K^{-}$and $K^{0} \bar{K}^{0}$ thresholds, in the $p p \rightarrow$ $p p K^{+} K^{-}$and $p n \rightarrow d K^{+} K^{-}$data. This finding has been attributed to $K^{+} K^{-} \rightleftharpoons K^{0} \bar{K}^{0}$ chargeexchange scattering ${ }^{[29]}$. This analysis also suggests that isospin-zero $K \bar{K}$ production is dominant. Independent of assumptions about the the details of the intermediate states, a re-analysis ${ }^{[30]}$ of the COSY$11 p p \rightarrow p p K^{+} K^{-}$data yields an estimate of the $K \bar{K}$ scattering length: $\left|\operatorname{Re}\left(a_{K^{+} K^{-}}\right)\right|=0.5_{-0.5}^{+4.0} \mathrm{fm}$ and $\operatorname{Im}\left(a_{K}^{+} K^{-}\right)=3.0 \pm 3.0 \mathrm{fm}$.

MOMO measured the $p d \rightarrow{ }^{3} \mathrm{He} K^{+} K^{-}$reaction at three excess energies, $\varepsilon=35,41$ and $55 \mathrm{MeV}^{[31]}$. Since the signs of the charges of the kaons were not identified, these data cannot be used to reliably isolate the $\bar{K}^{3} \mathrm{He}$ FSI ${ }^{[32]}$. Also, only upper limits for the $a_{0}(980)$ and $f_{0}(980)$ contributions of $25 \%$ and $10 \%$ could be determined ${ }^{[32]}$ while there is a clear peak in the $K^{+} K^{-}$invariant mass distributions that can be attributed to a strong $\phi(1020)$ contribution..

The total $d d \rightarrow{ }^{4} \mathrm{He} K^{+} K^{-}$cross section has been measured at ANKE ${ }^{[33]}$. This reaction is of particular interest since a $d d \rightarrow{ }^{4} \mathrm{He} X$ reaction may serve as a filter for isospin-zero states $X$. However, as seen

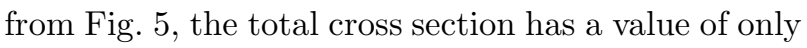
$5 \mathrm{pb}$, which is several orders below that of all other reactions. With such a low value, this is the rarest reaction that has so far been measured at COSY. It should be noted that the total $d d$ cross section at these energies is about eleven orders of magnitude larger.

To summarize, there is ample evidence that the antikaon is strongly attracted to the recoil nucleons in $p N \rightarrow(2 N) K^{+} K^{-}$reactions. Only residual effects of the $K^{+} K^{-}$interaction are seen, including a cusp at the $K^{0} \bar{K}^{0}$ threshold. In order to better understand the dominating $\bar{K} N$ FSI and, in particular, the role of possible intermediate $\Sigma(1385)$ and $\Lambda(1405)$ formation a combined analysis of their strange and nonstrange $\left.{ }^{[35,}, 36\right]$ decays has been suggested ${ }^{[34]}$.

In order better to restrict the contribution of the $a_{0} / f_{0}$ resonances to the $p d \rightarrow{ }^{3} \mathrm{He} K^{+} K^{-}$reaction, the WASA collaboration has measured the $p d \rightarrow{ }^{3} \mathrm{He} \eta \pi^{0} / \pi^{0} \pi^{0}$ processes. The data, which are currently being analyzed, will yield information about the $p d \rightarrow{ }^{3} \mathrm{He} a_{0} / f_{0}$ cross sections from the detection of the non-strange strong $a_{0} \rightarrow \eta \pi^{0}$ and $f_{0} \rightarrow \pi^{0} \pi^{0}$ decays. One may hope that either these mesonic excitations dominate or that they are negligibly small. In either case the analysis of the $K \bar{K}$ data would then be simplified since only one type of production mech- 
anism (viz. scalar mesons or hyperons) would need to be considered.

The author is grateful to P. Goslawski, A. Kupść and $C$. Wilkin for stimulating discussions during the preparation of the manuscript.

\section{References}

1 R. Maier et al., Nucl. Instrum. Methods Phys. Res. Sect. A 390 (1997) 1.

2 S. Barsov et al., Nucl. Instrum. Methods Phys. Res. Sect. A 462 (2001) 354.

3 A. Khoukaz et al., Eur. Phys. J. D 5 (1999) 275.

4 M. Buescher et al., Nucl. Instrum. Meth. A 481 (2002) 378.

5 M. Hartmann et al. [ANKE Collaboration], Int. J. Mod. Phys. A 22 (2007) 317.

6 S. Dymov et al., Part. Nucl. Lett. 1 (2004) 40.

7 S. Abd El-Samad et al. [COSY-TOF Collaboration], Eur. Phys. J. A 42 (2009) 159 arXiv:0906.3095 [nucl-ex]].

8 S. Abdel-Samad et al. [COSY-TOF Collaboration], Phys. Lett. B 632 (2006) 27.

9 C. Bargholtz et al. [CELSIUS/WASA Collaboration], Nucl. Instrum. Meth. A 594 (2008) 339 arXiv:0803.2657 [nuclex]].

10 H. H. Adam et al. [WASA-at-COSY Collaboration], arXiv:nucl-ex/0411038.

11 T. Mersmann et al., Phys. Rev. Lett. 98 (2007) 242301.

12 P. Goslawski et al., arXiv:0908.3103 [physics.acc-ph].

13 C. Amsler et al. [Particle Data Group], Phys. Lett. B 667 (2008) 1.

14 M. Altmeier et al., Eur. Phys. J. A 23 (2005) 351, and references therein.

15 M. Bashkanov et al., Phys. Rev. C 76 (2007) 048201 arXiv:0708.2014 [nucl-ex]].

16 C. Bargholtz et al. [CELSIUS-WASA Collaboration], Phys. Lett. B 644 (2007) 299 arXiv:hep-ex/0609007.

17 M. Berlowski et al., Phys. Rev. D 77 (2008) 032004

18 C. Adolph et al. [WASA-at-COSY Collaboration], Phys. Lett. B 677 (2009) 24 arXiv:0811.2763 [nucl-ex]].

19 A. E. Dorokhov and M. A. Ivanov, Phys. Rev. D 75 (2007) 114007 arXiv:0704.3498 [hep-ph]].
20 C. Quentmeier et al., Phys. Lett. B 515 (2001) 276 arXiv:nucl-ex/0103001.

21 P. Winter et al., Phys. Lett. B 635 (2006) 23 arXiv:hep-ex/0602030.

22 M. Hartmann et al., Phys. Rev. Lett. 96 (2006) 242301 [Erratum-ibid. 97 (2006) 029901] arXiv:hep-ex/0604010].

23 Y. Maeda et al. [The ANKE Collaboration], Phys. Rev. C 77 (2008) 015204 arXiv:0710.1755 [nucl-ex]].

24 V. Kleber et al., Phys. Rev. Lett. 91 (2003) 172304 arXiv:nucl-ex/0304020.

25 A. Dzyuba et al., Eur. Phys. J. A 29 (2006) 245 arXiv:nucl-ex/0605030.

26 Y. Maeda et al., Phys. Rev. Lett. 97 (2006) 142301 arXiv:nucl-ex/0607001.

27 Y. Maeda et al., Phys. Rev. C $\mathbf{7 9}$ (2009) 018201 arXiv:0811.4303 [nucl-ex]].

28 A. Dzyuba, M. Buscher, C. Hanhart, V. Kleber, V. Koptev, H. Stroher and C. Wilkin, Eur. Phys. J. A 38 (2008) 1 arXiv:0804.3695 [nucl-ex]].

29 A. Dzyuba et al., Phys. Lett. B 668 (2008) 315 arXiv:0807.0524 [nucl-th]].

30 M. Silarski et al., Phys. Rev. C 80 (2009) 045202 arXiv:0909.3974 [hep-ph]].

31 F. Bellemann et al. [COSY-MOMO Collaboration], Phys. Rev. C 75 (2007) 015204 arXiv:nucl-ex/0608047.

32 V. Y. Grishina, M. Buscher and L. A. Kondratyuk, Phys. Rev. C 75, 015208 (2007) arXiv:nucl-th/0608072.

33 X. Yuan et al., Eur. Phys. J. A 42 (2009) 1 arXiv:0905.0979 [nucl-ex]].

34 C. Wilkin, Acta Phys. Polon. Supp. 2 (2009) 89 arXiv:0812.0098 [nucl-th]]

35 I. Zychor et al., Phys. Lett. B 660 (2008) 167 arXiv:0705.1039 [nucl-ex]].

36 I. Zychor et al., Phys. Rev. Lett. 96 (2006) 012002 arXiv:nucl-ex/0506014. 